\title{
Viscosity Measurements on Gaseous Methane: Re-evaluation
}

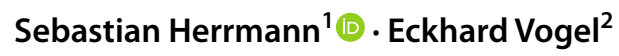

Received: 8 February 2022 / Accepted: 10 February 2022 / Published online: 28 February 2022

(c) The Author(s) 2022

\begin{abstract}
Previous experimental viscosity data for gaseous methane, published by Schley et al. (Int J Thermophys 25:1623, 2004) and originally obtained using a vibrating-wire viscometer in the temperature range between $260 \mathrm{~K}$ and $360 \mathrm{~K}$, were re-evaluated after an improved re-calibration. For this purpose, a new reference value for argon at 298.15 K and at zero density, proposed by Vogel et al. (Mol Phys 108:3335, 2010) and further updated by Hellmann (Private Communication, 2020), was applied.
\end{abstract}

Keywords Methane $\cdot$ Re-evaluation · Viscosity

Previous measurements of the viscosity $\eta$ for methane, carried out by Schley et al. [1] using a vibrating-wire viscometer with freely suspended weight and additional measurements of temperature $T$ and pressure $p$ for calculating the required density $\rho$ with an equation of state by Setzmann, Wagner, and de Reuck [2, 3], have been reevaluated. The re-evaluation concerns the specification of the wire radius by means of an improved calibration. The re-evaluated data, given as $\eta \rho p T$ values, are to be used together with other new accurate $\eta \rho p T$ data to generate a prospective viscosity correlation for methane.

The re-calibration of the applied vibrating-wire viscometer was performed in that way that the radius of the wire was newly specified using previous measurements on argon [4]. The original calibration employed an experimentally based reference value of Kestin and Leidenfrost [5] nowadays considered as obsolete. For the re-calibration, we used a today accepted value for the zero-density viscosity coefficient of argon, derived by Vogel et al. [6] from an ab initio potential on the basis of the kinetic theory of dilute gases and upgraded by Hellmann [7], to

Sebastian Herrmann

s.herrmann@hszg.de

1 Fakultät Maschinenwesen, Hochschule Zittau/Görlitz, 02763 Zittau, Germany

2 Institut für Chemie, Universität Rostock, 18059 Rostock, Germany 
be $\eta_{0, \mathrm{Ar}, 298.15 \mathrm{~K}}=22.5534 \mu \mathrm{Pa} \cdot \mathrm{s}$ with a standard uncertainty of $\pm 0.07 \%$. The wire radius amounts to $12.7548 \mu \mathrm{m}$ using the new reference value for argon.

The results reported in Table II of the previous paper of Schley et al. [1] were restricted to $\eta \rho p$ triples along the measured isotherms. In this new report, we include more details in order to make the information comparable to that given for the new viscosity measurements by Humberg et al. [8]. They recommended further a re-evaluation of the measurements by Schley et al. The individual points were not exactly measured at the nominal temperature of an isotherm $T_{\text {nom }}$, but could be kept within small deviations from the nominal temperature each. The experimental viscosity data were adjusted to $\eta_{T_{\text {nom }}}$ values at the nominal temperature using a Taylor series expansion restricted to the first power in temperature.

For this, the experimentally determined value of the initial density dependence $(\partial \eta / \partial T)_{\rho}=(0.030$ to 0.034$) \mu \mathrm{Pa} \cdot \mathrm{s} \cdot \mathrm{K}^{-1}$ advised by Vogel [9] for methane was used. Further, it is supposed that the density values $\rho_{\operatorname{eos}(T, p)}$, computed from the measured data for $T$ and $p$ using the equation of state by Setzmann, Wagner, and de Reuck [2, 3], and those for the isotherms are the same. As a result of this, the pressures $p_{T_{\text {nom }}, \rho_{\text {eos }}}$ at the nominal temperature changed and were recalculated from the densities. The improved experimental $\eta \rho p T$ data of this work for the previous measurements of Schley et al. [1] on methane (six isotherms at $260 \mathrm{~K}$, $280 \mathrm{~K}, 300 \mathrm{~K}, 320 \mathrm{~K}, 340 \mathrm{~K}$, and $360 \mathrm{~K}$ ) are summarized in Tables 1, 2, 3, 4, 5 , and 6 , in which the data are given in the sequential arrangement of the original measurements.

The experimental data of each nominal isotherm for methane were correlated as a function of the reduced density $\delta$ by means of a power-series representation restricted to the fourth power:

$$
\begin{aligned}
\eta(\tau, \delta) & =\sum_{i=0}^{4} \eta_{i}(\tau) \delta^{i}, \quad \delta=\frac{\rho}{\rho_{\mathrm{c}, \mathrm{CH}_{4}}}, \quad \tau=\frac{T}{T_{\mathrm{c}, \mathrm{CH}_{4}}}, \\
\text { with } \rho_{\mathrm{c}, \mathrm{CH}_{4}} & =162.66 \mathrm{~kg} \cdot \mathrm{m}^{-3}, T_{\mathrm{c}, \mathrm{CH}_{4}}=190.564 \mathrm{~K} .
\end{aligned}
$$

Here, $\delta$ is the reduced density, whereas $\tau$ is the reduced temperature. The values of the critical density $\rho_{\mathrm{c}, \mathrm{CH}_{4}}$ and of the critical temperature $T_{\mathrm{c}, \mathrm{CH}_{4}}$ are those given by Setzmann, Wagner, and de Reuck [2, 3]. Weighting factors $w_{i}=100 \eta_{\exp , i}^{-2}$ were used in the multiple linear least-squares regression to minimize the weighted sum of squares $\sigma=\sum_{i} w_{i}\left(\eta_{\mathrm{cor}, i}-\eta_{\exp , i}\right)^{2}$ as criterion for the quality of the representation of the considered isotherm. The coefficients $\eta_{i}(\tau)$ of Eq. 1 including their standard deviations s.d. $\eta_{i}$ and the weighted sum of squares $\sigma$ for each isotherm are given in Table 7. 
Table 1 Re-evaluated experimental $\eta \rho p T$ data for methane at $260 \mathrm{~K}$

\begin{tabular}{|c|c|c|c|c|c|}
\hline$T(\mathrm{~K})$ & $p(\mathrm{MPa})$ & $p_{260 \mathrm{~K}, \rho_{\cos }}(\mathrm{MPa})$ & $\rho_{\mathrm{eos}(T, p)}\left(\mathrm{kg} \cdot \mathrm{m}^{-3}\right)$ & $\eta(\mu \mathrm{Pa} \cdot \mathrm{s})$ & $\eta_{260 \mathrm{~K}}(\mu \mathrm{Pa} \cdot \mathrm{s})$ \\
\hline 260.13 & 19.854 & 19.825 & 205.16 & 22.620 & 22.615 \\
\hline 260.10 & 19.492 & 19.470 & 202.55 & 22.326 & 22.322 \\
\hline 260.07 & 19.007 & 18.992 & 198.92 & 21.910 & 21.908 \\
\hline 260.04 & 18.479 & 18.471 & 194.80 & 21.462 & 21.461 \\
\hline 260.01 & 17.991 & 17.989 & 190.81 & 21.029 & 21.029 \\
\hline 259.99 & 17.391 & 17.393 & 185.65 & 20.498 & 20.499 \\
\hline 259.97 & 17.018 & 17.023 & 182.32 & 20.159 & 20.160 \\
\hline 259.96 & 16.534 & 16.541 & 177.81 & 19.702 & 19.703 \\
\hline 259.96 & 16.002 & 16.009 & 172.61 & 19.208 & 19.209 \\
\hline 259.96 & 15.523 & 15.529 & 167.73 & 18.755 & 18.756 \\
\hline 259.96 & 15.003 & 15.009 & 162.23 & 18.261 & 18.262 \\
\hline 259.97 & 14.493 & 14.498 & 156.61 & 17.770 & 17.771 \\
\hline 259.97 & 13.988 & 13.992 & 150.85 & 17.297 & 17.298 \\
\hline 259.97 & 13.497 & 13.501 & 145.09 & 16.817 & 16.818 \\
\hline 259.97 & 13.013 & 13.017 & 139.25 & 16.369 & 16.370 \\
\hline 259.97 & 12.475 & 12.478 & 132.60 & 15.876 & 15.877 \\
\hline 259.98 & 12.011 & 12.013 & 126.76 & 15.456 & 15.457 \\
\hline 259.98 & 11.4752 & 11.4771 & 119.942 & 14.979 & 14.980 \\
\hline 259.98 & 11.0092 & 11.0110 & 113.968 & 14.572 & 14.573 \\
\hline 259.98 & 10.4966 & 10.4982 & 107.385 & 14.162 & 14.162 \\
\hline 259.99 & 9.9936 & 9.9944 & 100.939 & 13.769 & 13.769 \\
\hline 260.00 & 9.4550 & 9.4550 & 94.099 & 13.367 & 13.367 \\
\hline 260.09 & 9.0135 & 9.0077 & 88.499 & 13.054 & 13.051 \\
\hline 260.07 & 8.4848 & 8.4807 & 82.010 & 12.708 & 12.705 \\
\hline 260.06 & 7.9975 & 7.9943 & 76.144 & 12.410 & 12.408 \\
\hline 260.04 & 7.5015 & 7.4996 & 70.312 & 12.123 & 12.122 \\
\hline 260.02 & 6.9988 & 6.9980 & 64.550 & 11.8522 & 11.8515 \\
\hline 260.00 & 6.5024 & 6.5024 & 59.013 & 11.6044 & 11.6044 \\
\hline 259.99 & 5.9959 & 5.9962 & 53.520 & 11.3753 & 11.3756 \\
\hline 259.97 & 5.4974 & 5.4983 & 48.279 & 11.1632 & 11.1643 \\
\hline 259.96 & 4.9940 & 4.9951 & 43.144 & 10.9688 & 10.9701 \\
\hline 259.96 & 4.7449 & 4.7459 & 40.661 & 10.8774 & 10.8787 \\
\hline 259.96 & 4.4832 & 4.4841 & 38.094 & 10.7878 & 10.7892 \\
\hline 259.97 & 4.2613 & 4.2620 & 35.949 & 10.7148 & 10.7159 \\
\hline 259.97 & 3.9886 & 3.9893 & 33.356 & 10.6234 & 10.6245 \\
\hline 259.97 & 3.7393 & 3.7399 & 31.024 & 10.5494 & 10.5504 \\
\hline 259.97 & 3.4825 & 3.4829 & 28.659 & 10.4806 & 10.4816 \\
\hline 259.97 & 3.2477 & 3.2482 & 26.532 & 10.4108 & 10.4119 \\
\hline 259.96 & 2.9859 & 2.9865 & 24.196 & 10.3439 & 10.3453 \\
\hline 259.97 & 2.7329 & 2.7332 & 21.972 & 10.2837 & 10.2847 \\
\hline 259.97 & 2.5032 & 2.5036 & 19.985 & 10.2308 & 10.2318 \\
\hline 259.97 & 2.2622 & 2.2625 & 17.929 & 10.1784 & 10.1794 \\
\hline
\end{tabular}


Table 1 (continued)

\begin{tabular}{llllll}
\hline$T(\mathrm{~K})$ & $p(\mathrm{MPa})$ & $p_{260 \mathrm{~K}, \rho_{\cos }}(\mathrm{MPa})$ & $\rho_{\operatorname{eos}(T, p)}\left(\mathrm{kg} \cdot \mathrm{m}^{-3}\right)$ & $\eta(\mu \mathrm{Pa} \cdot \mathrm{s})$ & $\eta_{260 \mathrm{~K}}(\mu \mathrm{Pa} \cdot \mathrm{s})$ \\
\hline 259.97 & 1.9976 & 1.9979 & 15.707 & 10.1176 & 10.1186 \\
259.97 & 1.7530 & 1.7532 & 13.683 & 10.0703 & 10.0714 \\
259.97 & 1.5006 & 1.5008 & 11.6262 & 10.0250 & 10.0261 \\
260.11 & 1.2526 & 1.2521 & 9.6289 & 9.9870 & 9.9833 \\
260.11 & 1.00295 & 1.00250 & 7.6539 & 9.9406 & 9.9369 \\
260.11 & 0.89975 & 0.89934 & 6.8461 & 9.9258 & 9.9220 \\
260.11 & 0.80037 & 0.80007 & 6.0726 & 9.9111 & 9.9073 \\
260.11 & 0.70073 & 0.70036 & 5.3015 & 9.8932 & 9.8895 \\
260.11 & 0.60083 & 0.60060 & 4.5327 & 9.8833 & 9.8795 \\
260.12 & 0.49743 & 0.49725 & 3.7415 & 9.8665 & 9.8625 \\
260.12 & 0.39841 & 0.39817 & 2.9884 & 9.8524 & 9.8484 \\
260.12 & 0.30137 & 0.30118 & 2.2544 & 9.8381 & 9.8341 \\
260.11 & 0.20117 & 0.20113 & 1.5007 & 9.8227 & 9.8189 \\
260.12 & 0.10188 & 0.10185 & 0.75786 & 9.8026 & 9.7985 \\
\hline
\end{tabular}


Table 2 Re-evaluated experimental $\eta \rho p T$ data for methane at $280 \mathrm{~K}$

\begin{tabular}{|c|c|c|c|c|c|}
\hline$T(\mathrm{~K})$ & $p(\mathrm{MPa})$ & $p_{280 \mathrm{~K}, \rho_{\mathrm{cos}}}(\mathrm{MPa})$ & $\rho_{\mathrm{eos}(T, p)}\left(\mathrm{kg} \cdot \mathrm{m}^{-3}\right)$ & $\eta(\mu \mathrm{Pa} \cdot \mathrm{s})$ & $\eta_{280 \mathrm{~K}}(\mu \mathrm{Pa} \cdot \mathrm{s})$ \\
\hline 280.00 & 19.837 & 19.837 & 176.38 & 20.236 & 20.236 \\
\hline 280.01 & 19.469 & 19.467 & 173.62 & 19.975 & 19.974 \\
\hline 280.02 & 19.016 & 19.013 & 170.14 & 19.655 & 19.654 \\
\hline 280.02 & 18.514 & 18.511 & 166.19 & 19.301 & 19.300 \\
\hline 280.02 & 17.996 & 17.993 & 161.99 & 18.931 & 18.931 \\
\hline 280.01 & 17.504 & 17.503 & 157.90 & 18.567 & 18.566 \\
\hline 280.01 & 17.005 & 17.004 & 153.63 & 18.219 & 18.218 \\
\hline 280.01 & 16.510 & 16.509 & 149.28 & 17.849 & 17.849 \\
\hline 280.00 & 16.005 & 16.005 & 144.74 & 17.495 & 17.495 \\
\hline 280.00 & 15.504 & 15.504 & 140.13 & 17.140 & 17.140 \\
\hline 279.99 & 15.003 & 15.004 & 135.43 & 16.780 & 16.780 \\
\hline 280.06 & 14.481 & 14.475 & 130.37 & 16.407 & 16.405 \\
\hline 280.08 & 13.943 & 13.934 & 125.10 & 16.036 & 16.033 \\
\hline 280.07 & 13.505 & 13.498 & 120.79 & 15.744 & 15.741 \\
\hline 280.05 & 13.006 & 13.001 & 115.838 & 15.417 & 15.415 \\
\hline 280.03 & 12.485 & 12.483 & 110.624 & 15.075 & 15.074 \\
\hline 280.01 & 12.000 & 11.9991 & 105.734 & 14.772 & 14.771 \\
\hline 279.98 & 11.4938 & 11.4953 & 100.625 & 14.458 & 14.459 \\
\hline 279.96 & 11.0031 & 11.0059 & 95.662 & 14.167 & 14.168 \\
\hline 279.94 & 10.4991 & 10.5031 & 90.573 & 13.878 & 13.880 \\
\hline 279.91 & 9.9988 & 10.0044 & 85.551 & 13.601 & 13.604 \\
\hline 279.90 & 9.4946 & 9.5003 & 80.512 & 13.336 & 13.340 \\
\hline 279.88 & 8.9919 & 8.9982 & 75.540 & 13.080 & 13.084 \\
\hline 279.89 & 8.5157 & 8.5210 & 70.868 & 12.851 & 12.854 \\
\hline 279.88 & 7.9776 & 7.9829 & 65.670 & 12.603 & 12.607 \\
\hline 279.88 & 7.4998 & 7.5047 & 61.121 & 12.397 & 12.401 \\
\hline 279.88 & 7.0039 & 7.0083 & 56.475 & 12.194 & 12.198 \\
\hline 279.88 & 6.5016 & 6.5055 & 51.851 & 12.004 & 12.008 \\
\hline 279.89 & 5.9960 & 5.9993 & 47.283 & 11.8222 & 11.8259 \\
\hline 279.90 & 5.4944 & 5.4971 & 42.840 & 11.6536 & 11.6569 \\
\hline 279.91 & 4.9837 & 4.9858 & 38.410 & 11.4929 & 11.4958 \\
\hline 279.93 & 4.7424 & 4.7439 & 36.347 & 11.4196 & 11.4219 \\
\hline 279.95 & 4.4872 & 4.4882 & 34.189 & 11.3484 & 11.3501 \\
\hline 279.97 & 4.2319 & 4.2324 & 32.054 & 11.2760 & 11.2769 \\
\hline 279.99 & 3.9980 & 3.9981 & 30.119 & 11.2164 & 11.2167 \\
\hline 280.00 & 3.7364 & 3.7364 & 27.981 & 11.1446 & 11.1446 \\
\hline 280.01 & 3.4973 & 3.4971 & 26.047 & 11.0899 & 11.0896 \\
\hline 280.01 & 3.2471 & 3.2469 & 24.047 & 11.0308 & 11.0305 \\
\hline 280.02 & 2.9988 & 2.9985 & 22.083 & 10.9727 & 10.9721 \\
\hline 280.03 & 2.7428 & 2.7425 & 20.081 & 10.9225 & 10.9215 \\
\hline 280.04 & 2.4965 & 2.4961 & 18.176 & 10.8711 & 10.8698 \\
\hline 280.04 & 2.2264 & 2.2260 & 16.111 & 10.8162 & 10.8149 \\
\hline
\end{tabular}


Table 2 (continued)

\begin{tabular}{llllll}
\hline$T(\mathrm{~K})$ & $p(\mathrm{MPa})$ & $p_{280 \mathrm{~K}, \rho_{\mathrm{eos}}}(\mathrm{MPa})$ & $\rho_{\operatorname{eos}(T, p)}\left(\mathrm{kg} \cdot \mathrm{m}^{-3}\right)$ & $\eta(\mu \mathrm{Pa} \cdot \mathrm{s})$ & $\eta_{280 \mathrm{~K}}(\mu \mathrm{Pa} \cdot \mathrm{s})$ \\
\hline 280.03 & 1.9909 & 1.9906 & 14.332 & 10.7728 & 10.7718 \\
280.02 & 1.7459 & 1.7457 & 12.500 & 10.7254 & 10.7248 \\
280.02 & 1.4976 & 1.4975 & 10.6637 & 10.6821 & 10.6814 \\
280.01 & 1.2405 & 1.2404 & 8.7835 & 10.6391 & 10.6388 \\
280.00 & 0.99793 & 0.99789 & 7.0283 & 10.6023 & 10.6023 \\
280.00 & 0.90042 & 0.90043 & 6.3279 & 10.5874 & 10.5874 \\
280.00 & 0.79968 & 0.79975 & 5.6075 & 10.5717 & 10.5717 \\
279.99 & 0.70064 & 0.70073 & 4.9025 & 10.5570 & 10.5573 \\
279.99 & 0.60094 & 0.60100 & 4.1958 & 10.5466 & 10.5469 \\
279.99 & 0.49987 & 0.49983 & 3.4824 & 10.5300 & 10.5303 \\
279.99 & 0.40047 & 0.40050 & 2.7839 & 10.5194 & 10.5197 \\
279.99 & 0.30068 & 0.30074 & 2.0857 & 10.5018 & 10.5021 \\
279.99 & 0.19854 & 0.19853 & 1.3741 & 10.4868 & 10.4871 \\
279.99 & 0.10051 & 0.10049 & 0.69416 & 10.4666 & 10.4669 \\
\hline
\end{tabular}


Table 3 Re-evaluated experimental $\eta \rho p T$ data for methane at $300 \mathrm{~K}$

\begin{tabular}{|c|c|c|c|c|c|}
\hline$T(\mathrm{~K})$ & $p(\mathrm{MPa})$ & $p_{300 \mathrm{~K}, \rho_{\mathrm{cos}}}(\mathrm{MPa})$ & $\rho_{\operatorname{eos}(T, p)}\left(\mathrm{kg} \cdot \mathrm{m}^{-3}\right)$ & $\eta(\mu \mathrm{Pa} \cdot \mathrm{s})$ & $\eta_{300 \mathrm{~K}}(\mu \mathrm{Pa} \cdot \mathrm{s})$ \\
\hline 300.36 & 20.079 & 20.028 & 155.47 & 19.028 & 19.016 \\
\hline 300.35 & 19.572 & 19.525 & 151.95 & 18.735 & 18.723 \\
\hline 300.33 & 18.984 & 18.941 & 147.77 & 18.400 & 18.390 \\
\hline 300.31 & 18.512 & 18.473 & 144.35 & 18.131 & 18.121 \\
\hline 300.29 & 18.000 & 17.965 & 140.57 & 17.839 & 17.830 \\
\hline 300.28 & 17.508 & 17.476 & 136.86 & 17.560 & 17.551 \\
\hline 300.27 & 17.008 & 16.978 & 133.02 & 17.276 & 17.267 \\
\hline 300.26 & 16.499 & 16.471 & 129.05 & 16.983 & 16.974 \\
\hline 300.36 & 15.996 & 15.959 & 124.98 & 16.702 & 16.690 \\
\hline 300.34 & 15.512 & 15.479 & 121.12 & 16.441 & 16.430 \\
\hline 300.33 & 15.006 & 14.975 & 117.009 & 16.162 & 16.151 \\
\hline 300.32 & 14.545 & 14.517 & 113.233 & 15.923 & 15.912 \\
\hline 300.30 & 14.048 & 14.023 & 109.131 & 15.656 & 15.646 \\
\hline 300.28 & 13.479 & 13.457 & 104.400 & 15.364 & 15.355 \\
\hline 300.27 & 12.999 & 12.979 & 100.374 & 15.119 & 15.110 \\
\hline 300.27 & 12.519 & 12.500 & 96.330 & 14.882 & 14.873 \\
\hline 300.27 & 12.016 & 11.9977 & 92.073 & 14.642 & 14.634 \\
\hline 300.26 & 11.5266 & 11.5101 & 87.939 & 14.411 & 14.403 \\
\hline 300.25 & 10.9621 & 10.9474 & 83.171 & 14.157 & 14.149 \\
\hline 300.25 & 10.5010 & 10.4872 & 79.280 & 13.953 & 13.945 \\
\hline 300.24 & 9.9986 & 9.9862 & 75.059 & 13.740 & 13.732 \\
\hline 300.24 & 9.5112 & 9.4996 & 70.981 & 13.540 & 13.532 \\
\hline 300.23 & 9.0100 & 8.9997 & 66.817 & 13.342 & 13.335 \\
\hline 300.23 & 8.4735 & 8.4640 & 62.390 & 13.141 & 13.133 \\
\hline 300.36 & 7.9941 & 7.9804 & 58.430 & 12.969 & 12.958 \\
\hline 300.35 & 7.5080 & 7.4957 & 54.499 & 12.803 & 12.792 \\
\hline 300.34 & 7.0117 & 7.0008 & 50.528 & 12.638 & 12.627 \\
\hline 300.33 & 6.4804 & 6.4709 & 46.328 & 12.471 & 12.461 \\
\hline 300.32 & 5.9811 & 5.9728 & 42.431 & 12.322 & 12.312 \\
\hline 300.31 & 5.5018 & 5.4945 & 38.738 & 12.186 & 12.176 \\
\hline 300.30 & 5.0006 & 4.9944 & 34.929 & 12.053 & 12.043 \\
\hline 300.30 & 4.7591 & 4.7532 & 33.112 & 11.9902 & 11.9805 \\
\hline 300.30 & 4.5009 & 4.4954 & 31.184 & 11.9285 & 11.9189 \\
\hline 300.30 & 4.2541 & 4.2490 & 29.356 & 11.8682 & 11.8585 \\
\hline 300.31 & 3.9947 & 3.9898 & 27.447 & 11.8102 & 11.8002 \\
\hline 300.31 & 3.7562 & 3.7517 & 25.707 & 11.7569 & 11.7469 \\
\hline 300.31 & 3.5015 & 3.4972 & 23.862 & 11.7009 & 11.6910 \\
\hline 300.31 & 3.2523 & 3.2484 & 22.072 & 11.6473 & 11.6373 \\
\hline 300.31 & 2.9960 & 2.9925 & 20.246 & 11.5949 & 11.5849 \\
\hline 300.31 & 2.7408 & 2.7375 & 18.442 & 11.5479 & 11.5379 \\
\hline 300.31 & 2.4972 & 2.4944 & 16.735 & 11.5006 & 11.4906 \\
\hline 300.31 & 2.2534 & 2.2508 & 15.039 & 11.4581 & 11.4481 \\
\hline
\end{tabular}


Table 3 (continued)

\begin{tabular}{llllll}
\hline$T(\mathrm{~K})$ & $p(\mathrm{MPa})$ & $p_{300 \mathrm{~K}, \rho_{\cos }}(\mathrm{MPa})$ & $\rho_{\operatorname{eos}(T, p)}\left(\mathrm{kg} \cdot \mathrm{m}^{-3}\right)$ & $\eta(\mu \mathrm{Pa} \cdot \mathrm{s})$ & $\eta_{300 \mathrm{~K}}(\mu \mathrm{Pa} \cdot \mathrm{s})$ \\
\hline 300.31 & 2.0059 & 2.0037 & 13.332 & 11.4137 & 11.4037 \\
300.30 & 1.7534 & 1.7514 & 11.6043 & 11.3724 & 11.3627 \\
300.30 & 1.4930 & 1.4914 & 9.8376 & 11.3321 & 11.3224 \\
300.30 & 1.2532 & 1.2520 & 8.2245 & 11.2955 & 11.2859 \\
300.30 & 0.99661 & 0.99556 & 6.5121 & 11.2574 & 11.2477 \\
300.30 & 0.89862 & 0.89767 & 5.8620 & 11.2424 & 11.2327 \\
300.31 & 0.79654 & 0.79568 & 5.1870 & 11.2284 & 11.2184 \\
300.31 & 0.69734 & 0.69653 & 4.5334 & 11.2177 & 11.2077 \\
300.31 & 0.60073 & 0.60009 & 3.8990 & 11.2062 & 11.1962 \\
300.31 & 0.50093 & 0.50043 & 3.2458 & 11.1940 & 11.1840 \\
300.31 & 0.40241 & 0.40197 & 2.6031 & 11.1802 & 11.1702 \\
300.31 & 0.30029 & 0.29995 & 1.9392 & 11.1622 & 11.1522 \\
300.32 & 0.20117 & 0.20097 & 1.2968 & 11.1503 & 11.1400 \\
300.32 & 0.099667 & 0.099496 & 0.64141 & 11.1309 & 11.1206 \\
\hline
\end{tabular}


Table 4 Re-evaluated experimental $\eta \rho p T$ data for methane at $320 \mathrm{~K}$

\begin{tabular}{|c|c|c|c|c|c|}
\hline$T(\mathrm{~K})$ & $p(\mathrm{MPa})$ & $p_{320 \mathrm{~K}, \rho_{\mathrm{eos}}}(\mathrm{MPa})$ & $\rho_{\operatorname{eos}(T, p)}\left(\mathrm{kg} \cdot \mathrm{m}^{-3}\right)$ & $\eta(\mu \mathrm{Pa} \cdot \mathrm{s})$ & $\eta_{320 \mathrm{~K}}(\mu \mathrm{Pa} \cdot \mathrm{s})$ \\
\hline 320.06 & 19.936 & 19.929 & 137.78 & 18.266 & 18.264 \\
\hline 320.04 & 19.508 & 19.503 & 135.04 & 18.065 & 18.063 \\
\hline 320.02 & 19.019 & 19.017 & 131.86 & 17.832 & 17.831 \\
\hline 320.01 & 18.525 & 18.524 & 128.60 & 17.594 & 17.594 \\
\hline 320.00 & 18.011 & 18.011 & 125.15 & 17.357 & 17.357 \\
\hline 319.99 & 17.539 & 17.540 & 121.96 & 17.131 & 17.131 \\
\hline 319.98 & 17.033 & 17.035 & 118.488 & 16.900 & 16.901 \\
\hline 319.97 & 16.525 & 16.528 & 114.963 & 16.677 & 16.678 \\
\hline 319.97 & 16.017 & 16.020 & 111.394 & 16.437 & 16.438 \\
\hline 319.96 & 15.495 & 15.498 & 107.700 & 16.205 & 16.206 \\
\hline 319.95 & 14.998 & 15.002 & 104.152 & 15.983 & 15.984 \\
\hline 319.94 & 14.500 & 14.505 & 100.573 & 15.775 & 15.777 \\
\hline 319.96 & 14.000 & 14.003 & 96.939 & 15.563 & 15.564 \\
\hline 319.95 & 13.493 & 13.496 & 93.254 & 15.353 & 15.355 \\
\hline 319.95 & 12.977 & 12.980 & 89.484 & 15.142 & 15.144 \\
\hline 319.95 & 12.482 & 12.485 & 85.852 & 14.940 & 14.942 \\
\hline 320.08 & 11.8703 & 11.8657 & 81.305 & 14.712 & 14.710 \\
\hline 320.07 & 11.5003 & 11.4964 & 78.591 & 14.565 & 14.563 \\
\hline 320.06 & 11.0000 & 10.9969 & 74.921 & 14.381 & 14.379 \\
\hline 320.05 & 10.4933 & 10.4909 & 71.208 & 14.201 & 14.199 \\
\hline 320.03 & 10.0023 & 10.0009 & 67.621 & 14.030 & 14.029 \\
\hline 320.02 & 9.4991 & 9.4983 & 63.952 & 13.858 & 13.858 \\
\hline 320.01 & 8.9947 & 8.9943 & 60.289 & 13.693 & 13.693 \\
\hline 320.00 & 8.4947 & 8.4947 & 56.676 & 13.538 & 13.538 \\
\hline 319.99 & 8.0047 & 8.0050 & 53.155 & 13.389 & 13.389 \\
\hline 319.98 & 7.4801 & 7.4807 & 49.410 & 13.235 & 13.236 \\
\hline 319.97 & 6.9969 & 6.9977 & 45.986 & 13.103 & 13.104 \\
\hline 319.96 & 6.4848 & 6.4859 & 42.386 & 12.965 & 12.966 \\
\hline 319.95 & 5.9993 & 6.0005 & 39.002 & 12.837 & 12.839 \\
\hline 319.95 & 5.4892 & 5.4902 & 35.477 & 12.713 & 12.714 \\
\hline 319.94 & 4.9997 & 5.0008 & 32.130 & 12.599 & 12.600 \\
\hline 319.94 & 4.7526 & 4.7538 & 30.453 & 12.543 & 12.544 \\
\hline 319.95 & 4.5079 & 4.5087 & 28.798 & 12.492 & 12.493 \\
\hline 319.96 & 4.2485 & 4.2491 & 27.055 & 12.434 & 12.436 \\
\hline 319.96 & 4.0085 & 4.0091 & 25.452 & 12.385 & 12.387 \\
\hline 319.96 & 3.7591 & 3.7597 & 23.795 & 12.333 & 12.334 \\
\hline 319.97 & 3.4921 & 3.4925 & 22.031 & 12.283 & 12.284 \\
\hline 319.97 & 3.2600 & 3.2604 & 20.507 & 12.240 & 12.241 \\
\hline 319.97 & 2.9995 & 2.9997 & 18.806 & 12.190 & 12.191 \\
\hline 319.98 & 2.7428 & 2.7431 & 17.141 & 12.147 & 12.148 \\
\hline 319.98 & 2.4932 & 2.4933 & 15.531 & 12.103 & 12.104 \\
\hline 319.98 & 2.2531 & 2.2533 & 13.993 & 12.064 & 12.065 \\
\hline
\end{tabular}


Table 4 (continued)

\begin{tabular}{llllll}
\hline$T(\mathrm{~K})$ & $p(\mathrm{MPa})$ & $p_{320 \mathrm{~K}, \rho_{\cos }}(\mathrm{MPa})$ & $\rho_{\operatorname{eos}(T, p)}\left(\mathrm{kg} \cdot \mathrm{m}^{-3}\right)$ & $\eta(\mu \mathrm{Pa} \cdot \mathrm{s})$ & $\eta_{320 \mathrm{~K}}(\mu \mathrm{Pa} \cdot \mathrm{s})$ \\
\hline 319.98 & 2.0005 & 2.0007 & 12.384 & 12.025 & 12.025 \\
319.98 & 1.7492 & 1.7492 & 10.7924 & 11.9839 & 11.9845 \\
319.98 & 1.5026 & 1.5026 & 9.2415 & 11.9473 & 11.9480 \\
319.98 & 1.2501 & 1.2501 & 7.6633 & 11.9125 & 11.9131 \\
319.98 & 1.00008 & 1.00021 & 6.1106 & 11.8788 & 11.8795 \\
320.06 & 0.90159 & 0.90137 & 5.5003 & 11.8655 & 11.8636 \\
320.06 & 0.80028 & 0.80017 & 4.8757 & 11.8545 & 11.8526 \\
320.06 & 0.69957 & 0.69952 & 4.2565 & 11.8418 & 11.8399 \\
320.06 & 0.60029 & 0.60023 & 3.6477 & 11.8293 & 11.8274 \\
320.06 & 0.50009 & 0.50003 & 3.0348 & 11.8181 & 11.8162 \\
320.05 & 0.40013 & 0.40006 & 2.4251 & 11.8044 & 11.8028 \\
320.05 & 0.30001 & 0.29998 & 1.8159 & 11.7916 & 11.7901 \\
320.05 & 0.20142 & 0.20146 & 1.2175 & 11.7783 & 11.7767 \\
320.04 & 0.097179 & 0.097227 & 0.58664 & 11.7561 & 11.7549 \\
\hline
\end{tabular}


Table 5 Re-evaluated experimental $\eta \rho p T$ data for methane at $340 \mathrm{~K}$

\begin{tabular}{|c|c|c|c|c|c|}
\hline$T(\mathrm{~K})$ & $p(\mathrm{MPa})$ & $p_{340 \mathrm{~K}, \rho_{\mathrm{eos}}}(\mathrm{MPa})$ & $\rho_{\operatorname{eos}(T, p)}\left(\mathrm{kg} \cdot \mathrm{m}^{-3}\right)$ & $\eta(\mu \mathrm{Pa} \cdot \mathrm{s})$ & $\eta_{340 \mathrm{~K}}(\mu \mathrm{Pa} \cdot \mathrm{s})$ \\
\hline 340.04 & 28.773 & 28.767 & 170.47 & 21.614 & 21.612 \\
\hline 340.02 & 27.800 & 27.797 & 166.02 & 21.211 & 21.210 \\
\hline 340.01 & 26.949 & 26.948 & 162.00 & 20.855 & 20.855 \\
\hline 340.04 & 25.964 & 25.959 & 157.19 & 20.448 & 20.447 \\
\hline 340.02 & 24.966 & 24.963 & 152.19 & 20.023 & 20.022 \\
\hline 339.99 & 23.882 & 23.883 & 146.59 & 19.571 & 19.572 \\
\hline 339.97 & 22.945 & 22.949 & 141.60 & 19.188 & 19.189 \\
\hline 339.95 & 21.981 & 21.987 & 136.32 & 18.789 & 18.790 \\
\hline 339.93 & 20.982 & 20.990 & 130.70 & 18.378 & 18.380 \\
\hline 340.04 & 20.089 & 20.085 & 125.48 & 18.012 & 18.011 \\
\hline 340.02 & 19.023 & 19.021 & 119.187 & 17.584 & 17.583 \\
\hline 340.00 & 17.991 & 17.991 & 112.958 & 17.175 & 17.175 \\
\hline 339.97 & 17.019 & 17.022 & 106.987 & 16.798 & 16.799 \\
\hline 339.95 & 15.995 & 15.999 & 100.573 & 16.412 & 16.413 \\
\hline 339.92 & 15.002 & 15.008 & 94.274 & 16.048 & 16.051 \\
\hline 339.92 & 14.496 & 14.501 & 91.024 & 15.867 & 15.870 \\
\hline 339.94 & 14.010 & 14.014 & 87.890 & 15.695 & 15.697 \\
\hline 339.97 & 13.494 & 13.496 & 84.535 & 15.511 & 15.512 \\
\hline 339.98 & 12.971 & 12.972 & 81.138 & 15.331 & 15.331 \\
\hline 339.98 & 12.494 & 12.495 & 78.033 & 15.172 & 15.173 \\
\hline 339.98 & 11.8825 & 11.8835 & 74.042 & 14.970 & 14.971 \\
\hline 339.98 & 11.4908 & 11.4918 & 71.484 & 14.849 & 14.850 \\
\hline 339.98 & 10.9859 & 10.9868 & 68.186 & 14.691 & 14.691 \\
\hline 339.98 & 10.4666 & 10.4675 & 64.795 & 14.533 & 14.534 \\
\hline 339.98 & 9.9898 & 9.9906 & 61.686 & 14.390 & 14.391 \\
\hline 339.98 & 9.4977 & 9.4984 & 58.481 & 14.248 & 14.249 \\
\hline 339.98 & 9.0019 & 9.0026 & 55.260 & 14.109 & 14.110 \\
\hline 339.99 & 8.4909 & 8.4912 & 51.949 & 13.973 & 13.974 \\
\hline 340.00 & 8.0050 & 8.0050 & 48.812 & 13.847 & 13.847 \\
\hline 340.00 & 7.9406 & 7.9405 & 48.397 & 13.828 & 13.828 \\
\hline 340.00 & 7.4842 & 7.4843 & 45.467 & 13.715 & 13.715 \\
\hline 340.00 & 7.0060 & 7.0060 & 42.410 & 13.596 & 13.596 \\
\hline 340.00 & 6.4721 & 6.4720 & 39.015 & 13.470 & 13.470 \\
\hline 340.00 & 5.9912 & 5.9912 & 35.977 & 13.358 & 13.358 \\
\hline 340.00 & 5.5006 & 5.5007 & 32.897 & 13.254 & 13.254 \\
\hline 340.00 & 4.9156 & 4.9157 & 29.251 & 13.136 & 13.136 \\
\hline 340.00 & 4.7412 & 4.7411 & 28.169 & 13.100 & 13.100 \\
\hline 340.06 & 4.4865 & 4.4857 & 26.591 & 13.050 & 13.048 \\
\hline 340.06 & 4.2523 & 4.2515 & 25.150 & 13.007 & 13.005 \\
\hline 340.06 & 4.0025 & 4.0017 & 23.619 & 12.959 & 12.957 \\
\hline 340.05 & 3.7476 & 3.7469 & 22.063 & 12.913 & 12.911 \\
\hline 340.05 & 3.5061 & 3.5056 & 20.596 & 12.871 & 12.870 \\
\hline
\end{tabular}


Table 5 (continued)

\begin{tabular}{|c|c|c|c|c|c|}
\hline$T(\mathrm{~K})$ & $p(\mathrm{MPa})$ & $p_{340 \mathrm{~K}, \rho_{\mathrm{cos}}}(\mathrm{MPa})$ & $\rho_{\operatorname{eos}(T, p)}\left(\mathrm{kg} \cdot \mathrm{m}^{-3}\right)$ & $\eta(\mu \mathrm{Pa} \cdot \mathrm{s})$ & $\eta_{340 \mathrm{~K}}(\mu \mathrm{Pa} \cdot \mathrm{s})$ \\
\hline 340.04 & 3.2440 & 3.2436 & 19.010 & 12.825 & 12.824 \\
\hline 340.04 & 3.0040 & 3.0035 & 17.563 & 12.785 & 12.784 \\
\hline 340.04 & 2.7531 & 2.7527 & 16.058 & 12.744 & 12.743 \\
\hline 340.03 & 2.4972 & 2.4969 & 14.530 & 12.709 & 12.708 \\
\hline 340.03 & 2.2541 & 2.2539 & 13.085 & 12.671 & 12.670 \\
\hline 340.02 & 1.9946 & 1.9944 & 11.5491 & 12.635 & 12.634 \\
\hline 340.02 & 1.7492 & 1.7491 & 10.1040 & 12.597 & 12.596 \\
\hline 340.02 & 1.4925 & 1.4924 & 8.5991 & 12.565 & 12.564 \\
\hline 340.02 & 1.2636 & 1.2634 & 7.2635 & 12.538 & 12.538 \\
\hline 340.02 & 1.00122 & 1.00114 & 5.7401 & 12.502 & 12.502 \\
\hline 340.02 & 0.90554 & 0.90556 & 5.1866 & 12.490 & 12.489 \\
\hline 340.02 & 0.80276 & 0.80270 & 4.5931 & 12.476 & 12.476 \\
\hline 340.02 & 0.70130 & 0.70118 & 4.0084 & 12.465 & 12.465 \\
\hline 340.02 & 0.60212 & 0.60207 & 3.4380 & 12.453 & 12.452 \\
\hline 340.01 & 0.50192 & 0.50189 & 2.8631 & 12.442 & 12.441 \\
\hline 340.00 & 0.40440 & 0.40448 & 2.3045 & 12.432 & 12.432 \\
\hline 339.99 & 0.30053 & 0.30056 & 1.7109 & 12.419 & 12.419 \\
\hline 339.99 & 0.19776 & 0.19783 & 1.12459 & 12.401 & 12.401 \\
\hline 340.00 & 0.099605 & 0.099633 & 0.56584 & 12.383 & 12.383 \\
\hline
\end{tabular}


Table 6 Re-evaluated experimental $\eta \rho p T$ data for methane at $360 \mathrm{~K}$

\begin{tabular}{|c|c|c|c|c|c|}
\hline$T(\mathrm{~K})$ & $p(\mathrm{MPa})$ & $p_{360 \mathrm{~K}, \rho_{\mathrm{eos}}}(\mathrm{MPa})$ & $\rho_{\operatorname{eos}(T, p)}\left(\mathrm{kg} \cdot \mathrm{m}^{-3}\right)$ & $\eta(\mu \mathrm{Pa} \cdot \mathrm{s})$ & $\eta_{360 \mathrm{~K}}(\mu \mathrm{Pa} \cdot \mathrm{s})$ \\
\hline 360.01 & 29.185 & 29.183 & 158.93 & 21.191 & 21.191 \\
\hline 360.01 & 28.029 & 28.028 & 153.89 & 20.767 & 20.766 \\
\hline 360.02 & 26.906 & 26.903 & 148.82 & 20.348 & 20.347 \\
\hline 360.03 & 25.978 & 25.975 & 144.53 & 20.021 & 20.020 \\
\hline 360.03 & 24.996 & 24.993 & 139.87 & 19.659 & 19.658 \\
\hline 360.04 & 24.027 & 24.023 & 135.15 & 19.298 & 19.297 \\
\hline 360.04 & 22.887 & 22.883 & 129.46 & 18.900 & 18.899 \\
\hline 360.03 & 21.983 & 21.980 & 124.85 & 18.576 & 18.575 \\
\hline 360.03 & 20.985 & 20.982 & 119.640 & 18.220 & 18.219 \\
\hline 360.02 & 19.942 & 19.940 & 114.086 & 17.854 & 17.853 \\
\hline 360.01 & 18.990 & 18.989 & 108.918 & 17.522 & 17.522 \\
\hline 360.00 & 18.015 & 18.015 & 103.537 & 17.195 & 17.195 \\
\hline 359.99 & 17.036 & 17.036 & 98.044 & 16.869 & 16.869 \\
\hline 359.99 & 16.004 & 16.005 & 92.174 & 16.535 & 16.535 \\
\hline 359.99 & 14.993 & 14.994 & 86.356 & 16.214 & 16.215 \\
\hline 359.99 & 14.494 & 14.495 & 83.458 & 16.056 & 16.056 \\
\hline 359.98 & 13.983 & 13.984 & 80.480 & 15.901 & 15.901 \\
\hline 359.99 & 13.481 & 13.481 & 77.541 & 15.753 & 15.753 \\
\hline 359.99 & 12.985 & 12.986 & 74.635 & 15.607 & 15.607 \\
\hline 359.99 & 12.488 & 12.488 & 71.707 & 15.462 & 15.462 \\
\hline 360.00 & 11.9849 & 11.9849 & 68.740 & 15.322 & 15.322 \\
\hline 360.00 & 11.5041 & 11.5041 & 65.901 & 15.189 & 15.189 \\
\hline 360.00 & 10.9925 & 10.9925 & 62.878 & 15.051 & 15.051 \\
\hline 360.00 & 10.4821 & 10.4821 & 59.860 & 14.917 & 14.917 \\
\hline 359.98 & 9.9973 & 9.9980 & 56.998 & 14.790 & 14.791 \\
\hline 359.98 & 9.4823 & 9.4829 & 53.955 & 14.660 & 14.661 \\
\hline 359.98 & 8.9782 & 8.9788 & 50.980 & 14.537 & 14.538 \\
\hline 359.98 & 8.5100 & 8.5106 & 48.222 & 14.427 & 14.427 \\
\hline 359.97 & 8.0209 & 8.0218 & 45.348 & 14.309 & 14.310 \\
\hline 359.97 & 7.4950 & 7.4959 & 42.264 & 14.193 & 14.194 \\
\hline 359.98 & 7.0014 & 7.0018 & 39.376 & 14.089 & 14.090 \\
\hline 359.97 & 6.5000 & 6.5007 & 36.457 & 13.981 & 13.981 \\
\hline 359.97 & 6.0060 & 6.0065 & 33.590 & 13.882 & 13.883 \\
\hline 359.96 & 5.5031 & 5.5038 & 30.686 & 13.782 & 13.783 \\
\hline 359.96 & 5.0028 & 5.0035 & 27.810 & 13.692 & 13.693 \\
\hline 359.96 & 4.7515 & 4.7520 & 26.370 & 13.649 & 13.650 \\
\hline 359.96 & 4.5021 & 4.5027 & 24.946 & 13.600 & 13.602 \\
\hline 359.97 & 4.2508 & 4.2511 & 23.513 & 13.557 & 13.558 \\
\hline 359.98 & 3.9983 & 3.9986 & 22.079 & 13.517 & 13.517 \\
\hline 359.98 & 3.7513 & 3.7515 & 20.680 & 13.474 & 13.475 \\
\hline 359.99 & 3.5007 & 3.5007 & 19.264 & 13.432 & 13.433 \\
\hline 359.99 & 3.2510 & 3.2510 & 17.859 & 13.394 & 13.394 \\
\hline
\end{tabular}


Table 6 (continued)

\begin{tabular}{llllll}
\hline$T(\mathrm{~K})$ & $p(\mathrm{MPa})$ & $p_{360 \mathrm{~K}, \rho_{\mathrm{eos}}}(\mathrm{MPa})$ & $\rho_{\mathrm{eos}(T, p)}\left(\mathrm{kg} \cdot \mathrm{m}^{-3}\right)$ & $\eta(\mu \mathrm{Pa} \cdot \mathrm{s})$ & $\eta_{360 \mathrm{~K}}(\mu \mathrm{Pa} \cdot \mathrm{s})$ \\
\hline 359.98 & 3.0017 & 3.0018 & 16.461 & 13.355 & 13.356 \\
359.98 & 2.7436 & 2.7438 & 15.018 & 13.318 & 13.319 \\
359.98 & 2.4999 & 2.5001 & 13.660 & 13.289 & 13.289 \\
359.97 & 2.2470 & 2.2471 & 12.255 & 13.252 & 13.253 \\
359.97 & 1.9996 & 1.9998 & 10.886 & 13.216 & 13.217 \\
359.96 & 1.7506 & 1.7508 & 9.5131 & 13.184 & 13.186 \\
359.95 & 1.4988 & 1.4991 & 8.1296 & 13.154 & 13.155 \\
359.95 & 1.2512 & 1.2515 & 6.7736 & 13.122 & 13.123 \\
359.96 & 1.00063 & 1.00068 & 5.4064 & 13.092 & 13.093 \\
359.96 & 0.90014 & 0.90031 & 4.8596 & 13.077 & 13.078 \\
359.99 & 0.79961 & 0.79961 & 4.3131 & 13.071 & 13.071 \\
359.99 & 0.69939 & 0.69949 & 3.7695 & 13.058 & 13.058 \\
359.99 & 0.59989 & 0.59996 & 3.2307 & 13.046 & 13.046 \\
359.98 & 0.50016 & 0.50026 & 2.6916 & 13.036 & 13.037 \\
359.97 & 0.39974 & 0.39986 & 2.1495 & 13.021 & 13.022 \\
359.97 & 0.29870 & 0.29874 & 1.6049 & 13.009 & 13.010 \\
359.93 & 0.19670 & 0.19671 & 1.05611 & 12.994 & 12.997 \\
359.92 & 0.10261 & 0.10272 & 0.55056 & 12.972 & 12.974 \\
\hline & & & & & \\
\hline
\end{tabular}

Table 7 Coefficients of Eq. 1 for the re-evaluated viscosity measurements on methane

\begin{tabular}{llllll}
\hline$T(\mathrm{~K})$ & $n$ & $\rho_{\max }\left(\mathrm{kg} \cdot \mathrm{m}^{-3}\right)$ & \multicolumn{1}{l}{$\eta_{0}(\mu \mathrm{Pa} \cdot \mathrm{s})$} & $\eta_{1}(\mu \mathrm{Pa} \cdot \mathrm{s})$ & $\eta_{2}(\mu \mathrm{Pa} \cdot \mathrm{s})$ \\
\hline 260 & 4 & 205.16 & $9.796 \pm 0.001$ & $2.712 \pm 0.015$ & $7.028 \pm 0.060$ \\
280 & 4 & 176.38 & $10.465 \pm 0.001$ & $2.858 \pm 0.016$ & $6.863 \pm 0.070$ \\
300 & 4 & 155.47 & $11.120 \pm 0.001$ & $2.965 \pm 0.014$ & $6.724 \pm 0.070$ \\
320 & 4 & 137.78 & $11.755 \pm 0.001$ & $3.034 \pm 0.017$ & $6.804 \pm 0.096$ \\
340 & 4 & 170.47 & $12.386 \pm 0.001$ & $2.996 \pm 0.015$ & $6.995 \pm 0.069$ \\
360 & 4 & 158.93 & $12.979 \pm 0.001$ & $3.122 \pm 0.024$ & $6.557 \pm 0.119$ \\
\hline$T(\mathrm{~K})$ & & & $\eta_{3}(\mu \mathrm{Pa} \cdot \mathrm{s})$ & $\eta_{4}(\mu \mathrm{Pa} \cdot \mathrm{s})$ & $\sigma$ \\
\hline 260 & & $-2.586 \pm 0.080$ & $1.349 \pm 0.034$ & 0.023 \\
280 & & $-2.504 \pm 0.108$ & $1.299 \pm 0.053$ & 0.020 \\
300 & & $-2.514 \pm 0.122$ & $1.339 \pm 0.068$ & 0.015 \\
320 & & $-2.978 \pm 0.186$ & $1.691 \pm 0.115$ & 0.015 \\
340 & & $-3.144 \pm 0.111$ & $1.680 \pm 0.057$ & 0.017 \\
360 & & $-2.556 \pm 0.205$ & $1.409 \pm 0.113$ & 0.025 \\
\hline
\end{tabular}


Funding Open Access funding enabled and organized by Projekt DEAL.

\section{Declarations}

Conflict of interest The authors declare that they have no conflict of interest.

Open Access This article is licensed under a Creative Commons Attribution 4.0 International License, which permits use, sharing, adaptation, distribution and reproduction in any medium or format, as long as you give appropriate credit to the original author(s) and the source, provide a link to the Creative Commons licence, and indicate if changes were made. The images or other third party material in this article are included in the article's Creative Commons licence, unless indicated otherwise in a credit line to the material. If material is not included in the article's Creative Commons licence and your intended use is not permitted by statutory regulation or exceeds the permitted use, you will need to obtain permission directly from the copyright holder. To view a copy of this licence, visit http://creativecommons.org/licen ses/by/4.0/.

\section{References}

1. P. Schley, M. Jaeschke, C. Küchenmeister, E. Vogel, Int. J. Thermophys. 25, 1623 (2004)

2. U. Setzmann, W. Wagner, Int. J. Thermophys. 10, 1103 (1989)

3. W. Wagner, K.M. de Reuck, Methane. International Thermodynamic Tables of the Fluid State-13 (Blackwell Science, Oxford, 1996)

4. J. Wilhelm, E. Vogel, Int. J. Thermophys. 21, 301 (2000)

5. J. Kestin, W. Leidenfrost, Physica 25, 1033 (1959)

6. E. Vogel, B. Jäger, R. Hellmann, E. Bich, Mol. Phys. 108, 3335 (2010)

7. R. Hellmann, Private communication (Helmut-Schmidt University/University of the Federal Armed Forces Hamburg, Hamburg, Germany, 2020)

8. K. Humberg, M. Richter, J.P.M. Trusler, R. Span, J. Chem. Thermodyn. 147, 106104 (2020)

9. E. Vogel, Private communication (University of Rostock, Rostock, Germany, 2020)

Publisher's Note Springer Nature remains neutral with regard to jurisdictional claims in published maps and institutional affiliations. 